\title{
Delay-dependent exponential stability criteria for stochastic systems with polytopic-type uncertainties
}

\author{
Yumei LI ${ }^{1,2}$, Xinping GUAN ${ }^{2}$, Dan PENG ${ }^{2}$, Changchun HUA ${ }^{2}$, Xiaoyuan $\mathrm{LUO}^{2}$ \\ (1.Institute of Mathematics and System Science, Xinjiang University, Urumuqi Xinjiang 830046, China; \\ 2.Institute of Electrical Engineering, Yanshan University, Qinhuangdao Hebei 066004, China)
}

\begin{abstract}
This paper considers the problem of delay-dependent exponential stability in mean square for stochastic systems with polytopic-type uncertainties and time-varying delay. Applying the descriptor model transformation and introducing free weighting matrices, a new type of Lyapunov-Krasovskii functional is constructed based on linear matrix inequalities (LMIs), and some new delay-dependent criteria are obtained. These criteria include the delay-independent/ratedependent and delay-dependent/rate-independent exponential stability criteria. These new criteria are less conservative than existing ones. Numerical examples demonstrate that these new criteria are effective and are an improvement over existing ones.
\end{abstract}

Keywords: Stochastic system; Exponential stability in mean square; Time-varying state delay; Delay-dependent criteria; Linear matrix inequality (LMI)

\section{Introduction}

Recently, delay-dependent stability criteria for stochastic delay systems have attracted extensive attention (see [1 8]). In view of the robustness of stochastic stability, the linear and semilinear systems were studied in $[1,2]$, respectively. [3] investigated the stability of linear and semilinear stochastic differential equation by means of the exponential stability. Verriest [4] presented stability of linear stochastic differential equation via Riccati equations. Based on the LMI approach, [5 8] gave the delay-dependent robust stability criteria of uncertain stochastic systems, respectively. However, the criteria in [5] involved the parameterized model transformation. To determine the stability of system, [6] and [8] used some inequality constraint. [7] used a descriptor integral inequality constraint, and the criteria in $[7,8]$ with matrix constraint $P \leqslant \alpha I$ ( $\alpha>0$ is a scalar, $P$ is the product of Lyapunov matrix). These results show considerable conservativeness.

This paper presents some new delay-dependent exponential stability criteria for stochastic system with polytopictype uncertainties and time-varying delay. First, applying descriptor model transformation [9], we set descriptor stochastic system and construct a new type of Lyapunov-Krasovskii functional. Second, based on the idea of [10], some free weighting matrices are introduced to exclude constraint conditions in [6 8]. Finally, using LMI algorithm, we obtain delay-dependent and delay-independent exponential stability criteria for stochastic system with polytopic-type uncertainties and timevarying delay. These criteria include delay-dependent/rateindependent and delay-independent/rate-dependent exponential stability criteria. In contrast with the existing stability criteria, these new criteria are less conservative. Numerical simulation examples show that these results are effective and an improvement over existing ones.

For convenience, we adopt the following notations: $\operatorname{tr}(A)\left(A^{\mathrm{T}}\right)$ denotes trace(transpose) of the matrix $A ; A \geqslant 0$ $(A>0)$ denotes positive semidefinite (positive definite) matrix $A ; L_{F_{0}}^{2}\left([-\tau, 0] ; \mathbb{R}^{n}\right)$ is the family of $\mathbb{R}^{n}$-valued stochastic processes $\eta(s),-\tau \leqslant s \leqslant 0$ such that $\eta(s)$ is $\digamma_{0}-$ measurable for every second and $\int_{-\tau}^{0} E\|\eta(s)\|^{2} \mathrm{~d} s<$ $\infty$; and $E\{\cdot\}$ denotes mathematical expectation operator with respect to the given probability measure $P$.

\section{Preliminaries}

Consider the robust stability of system (1) with polytopictype uncertainties, that is, assume that system (1) has the following form:

$$
\begin{aligned}
& \mathrm{d} x(t)= {\left[A x(t)+A_{d} x(t-h(t))\right] \mathrm{d} t } \\
&+\left[C x(t)+C_{d} x(t-h(t))\right] \mathrm{d} \beta(t), \\
& x(t)=\varphi(t), \quad \forall t \in[-\tau, 0],
\end{aligned}
$$

where $x(t) \in \mathbb{R}^{n}$ is the state vector, the system matrices $A, A_{d}, C$, and $C_{d}$ are assumed to be uncertain but belong to a known convex compact set of polytopic type, namely

$$
\left(A, A_{d}, C, C_{d}\right) \in \Omega,
$$

where $\Omega$ is a given convex bounded polyhedral domain described by $q$ vertices as follows:

$$
\begin{gathered}
\Omega:=\left\{\left(A, A_{d}, C, C_{d}\right)=\sum_{k=1}^{q} \xi_{k}\left(A_{k}, A_{k d}, C_{k}, C_{k d}\right)\right. \\
\left.\xi_{k} \geqslant 0 ; \sum_{k=1}^{q} \xi_{k}=1\right\} .
\end{gathered}
$$

The time delay $h(t)$ is a time-varying continuous function that satisfies

$$
0 \leqslant h(t) \leqslant \tau
$$

Received 14 January 2008; revised 24 July 2008.

This work was supported by the National Natural Science Foundation of China (No.60525303, 60604004, 60704009) and Natural Science Foundation of Hebei Province, China (No.F2005000390, F2006000270). 
and

$$
\dot{h(t)} \leqslant \mu \leqslant 1 \text {, }
$$

where $\tau$ and $\mu$ are constants, $\varphi(t)$ is a continuous vectorvalued initial function, and $\varphi:=\{\varphi(s):-\tau \leqslant s \leqslant 0\} \in$ $L_{\digamma 0}^{2}\left([-\tau, 0], \mathbb{R}^{n}\right)$. It is well known that (1) has a unique solution, denoted by $x(t, \varphi)$, which is square integrable. So (1) admits a trivial solution $x(t, 0) \equiv 0 . A_{k}, A_{k d}, C_{k}, C_{k d}$ are known constant matrices with appropriate dimensions. The variables $\beta(t)$ are an $m$-dimensional Brownian motion defined on a complete probability space $(\Omega, \digamma, P)$ with a natural filtration $\left\{\digamma_{t}\right\}_{t \geqslant 0}$ (i.e., $\digamma_{t}=\sigma\{\varpi(s): 0 \leqslant s \leqslant t\}$ ).

Definition 1 System (1) is said to be exponentially stable in mean square if there exists a positive constant $\alpha_{0}$ such that

$$
\lim _{t \rightarrow \infty} \sup \frac{1}{t} \log E\|x(t)\|^{2} \leqslant-\alpha_{0} .
$$

\subsection{Delay-dependent robust exponential stability}

To discuss the stability of system (1), first, we introduce the descriptor system approach, set

$$
\begin{aligned}
& q(t)=A x(t)+A_{d} x(t-h(t)), \\
& g(t)=C x(t)+C_{d} x(t-h(t)) .
\end{aligned}
$$

Then system (1) becomes the following descriptor stochastic system

$$
\mathrm{d} x(t)=q(t) \mathrm{d} t+g(t) \mathrm{d} \beta(t) .
$$

Moreover, equations in (6) and (7) ensure the following zero equations

$$
\begin{aligned}
& 2\left[x^{\mathrm{T}}(t) N_{1}+x^{\mathrm{T}}(t-h(t)) N_{2}+q^{\mathrm{T}}(t) N_{3}+g^{\mathrm{T}}(t) N_{4}\right] \\
& \times\left[A x(t)+A_{d} x(t-h(t))-q(t)\right] \equiv 0, \\
& 2\left[x^{\mathrm{T}}(t) T_{1}+x^{\mathrm{T}}(t-h(t)) T_{2}+q^{\mathrm{T}}(t) T_{3}+g^{\mathrm{T}}(t) T_{4}\right] \\
& \times\left[C x(t)+C_{d} x(t-h(t))-g(t)\right] \equiv 0,
\end{aligned}
$$

where $N_{r}$ and $T_{r}(r=1,2,3,4)$ are appropriately dimensioned matrices. On the other hand, the Newton-Leibniz formula provides

$$
x(t)-x(t-h(t))=\int_{t-h(t)}^{t} \dot{x}(s) \mathrm{d} s=\int_{t-h(t)}^{t} q(s) \mathrm{d} s+\varsigma,
$$

where $\varsigma^{\mathrm{T}}=\left[\int_{t-h(t)}^{t} g(s) \mathrm{d} \beta(s)\right]^{\mathrm{T}}$ by (11), get

$$
\int_{t-h(t)}^{t} q(s) \mathrm{d} s=x(t)-x(t-h(t))-\varsigma,
$$

then, we obtained the following Theorem.

Theorem 1 Consider system (1) with polytopic-type uncertainties (3) and a time-varying delay satisfying (4) and (5). Given scalars $\tau>0$ and $\mu<1$, system (1) is robust exponentially stable in mean square, if there exist symmetric positive definite matrices $P_{k}>0, Q_{k} \geqslant 0, Z_{k}>0$, and $R_{i j}^{(k)}(i, j=1,2,3)$ and appropriately dimensioned matrices $N_{r}$ and $T_{r}(r=1,2,3,4)$ such that $R_{i j}^{(k)}=R_{j i}^{(k)}$ and the following LMIs hold for $k=1, \cdots, q$ :

$$
\Phi^{(k)}=\left[\begin{array}{ccccc}
\Phi_{11}^{(k)} & \Phi_{12}^{(k)} & \Phi_{13}^{(k)} & \Phi_{14}^{(k)} & R_{13}^{(k)} \\
* & \Phi_{22}^{(k)} & \Phi_{23}^{(k)} & \Phi_{24}^{(k)} & R_{23}^{(k)} \\
* & * & \Phi_{33}^{(k)} & \Phi_{34}^{(k)} & 0 \\
* & * & * & \Phi_{44}^{(k)} & 0 \\
* & * & * & * & -Z_{k}
\end{array}\right]<0
$$

and

$$
R^{(k)}=\left[\begin{array}{ccc}
R_{11}^{(k)} & R_{12}^{(k)} & R_{13}^{(k)} \\
* & R_{22}^{(k)} & R_{23}^{(k)} \\
* & * & R_{33}^{(k)}
\end{array}\right]>0,
$$

where an asterisk $*$ denotes a block induced easily by symmetry and

$$
\Phi_{11}^{(k)}=N_{1} A_{k}+A_{k}^{\mathrm{T}} N_{1}^{\mathrm{T}}+T_{1} C_{k}+C_{k}^{\mathrm{T}} T_{1}^{\mathrm{T}}+Q_{k}+\tau R_{11}^{(k)}
$$$$
+R_{13}^{(k)}+R_{13}^{(k) T} \text {, }
$$

$\Phi_{12}^{(k)}=N_{1} A_{d k}+A_{k}^{\mathrm{T}} N_{2}^{\mathrm{T}}+T_{1} C_{d k}+C_{k}^{\mathrm{T}} T_{2}^{\mathrm{T}}+\tau R_{12}^{(k)}$

$$
-R_{13}^{(k)}+R_{23}^{(k) T} \text {, }
$$

$\Phi_{13}^{(k)}=P_{k}-N_{1}+A_{k}^{\mathrm{T}} N_{3}^{\mathrm{T}}+C_{k}^{\mathrm{T}} T_{3}^{\mathrm{T}}$,

$\Phi_{14}^{(k)}=A_{k}^{\mathrm{T}} N_{4}^{\mathrm{T}}-T_{1}+C_{k}^{\mathrm{T}} T_{4}^{\mathrm{T}}$,

$\Phi_{22}^{(k)}=N_{2} A_{d k}+A_{d k}^{\mathrm{T}} N_{2}^{\mathrm{T}}+T_{2} C_{d k}-(1-\mu) Q_{k}+C_{d k}^{\mathrm{T}} T_{2}^{\mathrm{T}}$ $+\tau R_{22}^{(k)}-R_{23}^{(k)}-R_{23}^{(k) T}$,

$\Phi_{23}^{(k)}=-N_{2}+A_{d k}^{\mathrm{T}} N_{3}^{\mathrm{T}}+C_{d k}^{\mathrm{T}} T_{3}^{\mathrm{T}}$,

$\Phi_{24}^{(k)}=A_{d k}^{\mathrm{T}} N_{4}^{\mathrm{T}}-T_{2}+C_{d k}^{\mathrm{T}} T_{4}^{\mathrm{T}}$,

$\Phi_{33}^{(k)}=-N_{3}-N_{3}^{\mathrm{T}}+\tau R_{33}^{(k)}$,

$\Phi_{34}^{(k)}=-N_{4}^{\mathrm{T}}-T_{3}, \Phi_{44}^{(k)}=P_{k}-T_{4}^{\mathrm{T}}-T_{4}+\tau Z_{k}$.

Proof Choose a Lyapunov-Krasovskii functional for system (1) to be

$$
V(t)=\sum_{i=1}^{5} V_{i}(t)
$$

in which

$$
\begin{aligned}
& V_{1}(t)=\sum_{k=1}^{q} x(t)^{\mathrm{T}} P_{k} x(t), \\
& V_{2}(t)=\sum_{k=1}^{q} \int_{t-h(t)}^{t} x^{\mathrm{T}}(s) Q_{k} x(s) \mathrm{d} s, \\
& V_{3}(t)=\sum_{k=1}^{q} \int_{-\tau}^{0} \int_{t+\theta}^{t} q^{\mathrm{T}}(s) R_{33}^{(k)} q(s) \mathrm{d} s \mathrm{~d} \theta, \\
& V_{4}(t)=\sum_{k=1}^{q} \int_{-\tau}^{0} \int_{t+\theta}^{t} \operatorname{tr}\left[g^{\mathrm{T}}(s) Z_{k} g(s)\right] \mathrm{d} s \mathrm{~d} \theta, \\
& V_{5}(t)=\sum_{k=1}^{q} \int_{0}^{t} \int_{\alpha-h(\alpha)}^{\alpha} \delta^{\mathrm{T}} R_{k} \delta \mathrm{d} s \mathrm{~d} \alpha,
\end{aligned}
$$

where $\delta^{\mathrm{T}}=\left[x^{\mathrm{T}}(\alpha), x^{\mathrm{T}}(\alpha-h(\alpha)), q^{\mathrm{T}}(s)\right]$,

$$
R^{(k)}=\left[\begin{array}{ccc}
R_{11}^{(k)} & R_{12}^{(k)} & R_{13}^{(k)} \\
* & R_{22}^{(k)} & R_{23}^{(k)} \\
* & * & R_{33}^{(k)}
\end{array}\right] .
$$

$P_{k}, Q_{k}, Z_{k}, R_{i j}^{(k)}(i, j=1,2,3)$ are positive definite matrices with appropriate dimensions. Let $L$ be the weak infinitesimal operator of (8), then, by Itô differential formula,

$$
\begin{aligned}
L_{v=0} V_{1}= & \sum_{k=1}^{q}\left\{2 x^{\mathrm{T}}(t) P_{k} q(t)+\operatorname{tr}\left[g^{\mathrm{T}}(t) P_{k} g(t)\right]\right\}, \\
L_{v=0} V_{2} \leqslant & \sum_{k=1}^{q}\left\{x^{\mathrm{T}}(t) Q_{k} x(t)\right. \\
& \left.-(1-\mu) x^{\mathrm{T}}(t-h(t)) Q_{k} x(t-h(t))\right\}, \\
L_{v=0} V_{3} \leqslant & \sum_{k=1}^{q}\left\{\tau q^{\mathrm{T}}(t) R_{33}^{(k)} q(t)-\chi\right\}
\end{aligned}
$$




$$
\begin{aligned}
L_{v=0} V_{4} \leqslant & \sum_{k=1}^{q}\left\{\tau \operatorname{tr}\left[g^{\mathrm{T}}(t) Z_{k} g(t)\right]\right. \\
& \left.-\int_{t-h(t)}^{t} \operatorname{tr}\left[g^{\mathrm{T}}(s) Z_{k} g(s)\right] \mathrm{d} s\right\}, \\
L_{v=0} V_{5}= & \sum_{k=1}^{q}\left\{h(t) \xi^{\mathrm{T}}\left[\begin{array}{cc}
R_{11}^{(k)} & R_{12}^{(k)} \\
R_{12}^{(k) T} & R_{22}^{(k)}
\end{array}\right] \xi\right. \\
& \left.+2 \int_{t-h(t)}^{t} \xi^{\mathrm{T}}\left[\begin{array}{c}
R_{13}^{(k)} \\
R_{23}^{(k)}
\end{array}\right] q(s) \mathrm{d} s+\chi\right\},
\end{aligned}
$$

where $\chi=\int_{t-h(t)}^{t} q^{\mathrm{T}}(s) R_{33}^{(k)} q(s) \mathrm{d} s$.

Substitute (12) into (18), get

$$
\begin{aligned}
L_{v=0} V_{5} & \sum_{k=1}^{q}\left\{h(t) \xi^{\mathrm{T}}\left[\begin{array}{cc}
R_{11}^{(k)} & R_{12}^{(k)} \\
R_{12}^{(k) T} & R_{22}^{(k)}
\end{array}\right] \xi\right. \\
& \left.+2 \xi^{\mathrm{T}}\left[\begin{array}{c}
R_{13}^{(k)}-R_{13}^{(k)} \\
R_{23}^{(k)}-R_{23}^{(k)}
\end{array}\right] \xi+\chi-2 \xi^{\mathrm{T}}\left[\begin{array}{c}
R_{13}^{(k)} \\
R_{23}^{(k)}
\end{array}\right] \varsigma\right\} .
\end{aligned}
$$

By Lemma [11], for any matrix $Z_{k}>0$,

$$
-2 \xi^{\mathrm{T}}\left[\begin{array}{c}
R_{13}^{(k)} \\
R_{23}^{(k)}
\end{array}\right] \varsigma \leqslant \xi^{\mathrm{T}}\left[\begin{array}{c}
R_{13}^{(k)} \\
R_{23}^{(k)}
\end{array}\right] Z_{k}^{-1}\left[\begin{array}{c}
R_{13}^{(k)} \\
R_{23}^{(k)}
\end{array}\right]^{\mathrm{T}} \xi+\varsigma^{\mathrm{T}} Z_{k} \varsigma .
$$

Obviously,

$$
\begin{aligned}
& L_{v=0} V_{5} \\
& \leqslant \sum_{k=1}^{q}\left\{\tau \xi^{\mathrm{T}}\left[\begin{array}{cc}
R_{11}^{(k)} & R_{12}^{(k)} \\
R_{12}^{T(k)} & R_{22}^{(k)}
\end{array}\right] \xi+2 \xi^{\mathrm{T}}\left[\begin{array}{cc}
R_{13}^{(k)} & -R_{13}^{(k)} \\
R_{23}^{(k)} & -R_{23}^{(k)}
\end{array}\right] \xi\right. \\
& \left.\quad+\chi+\varsigma^{\mathrm{T}} Z_{k} \varsigma+\xi^{\mathrm{T}}\left[\begin{array}{c}
R_{13}^{(k)} \\
R_{23}^{(k)}
\end{array}\right] Z_{k}^{-1}\left[\begin{array}{c}
R_{13}^{(k)} \\
R_{23}^{(k)}
\end{array}\right]^{\mathrm{T}} \xi\right\},
\end{aligned}
$$

where $\xi^{\mathrm{T}}=\left[x^{\mathrm{T}}(t), x^{\mathrm{T}}(t-h(t))\right]$. Combining $L_{v=0} V_{i}$ ( $i=1,2,3,4,5)$ and adding the terms on the left of (9)(10) to $L_{v=0} V$, we can express $L_{v=0} V$ as

$$
\begin{aligned}
L_{v=0} V \leqslant & \sum_{k=1}^{q}\left\{\eta^{\mathrm{T}}(t) \xi_{k} \Phi^{(k)} \eta(t)\right. \\
& \left.-\int_{t-h(t)}^{t} \operatorname{tr}\left[g^{\mathrm{T}}(s) Z_{k} g(s)\right] \mathrm{d} s+\varsigma^{\mathrm{T}} Z_{k} \varsigma\right\}
\end{aligned}
$$

where $\eta^{\mathrm{T}}(t)=\left[x^{\mathrm{T}}(t), x^{\mathrm{T}}(t-h(t)), q^{\mathrm{T}}(t), g^{\mathrm{T}}(t)\right], \sigma^{\mathrm{T}}=$ $\left[\left(R_{13}^{(k)}\right)^{\mathrm{T}},\left(R_{23}^{(k)}\right)^{\mathrm{T}}, 0,0\right]$,

$$
\Phi^{(k)}=\left[\begin{array}{cccc}
\Phi_{11}^{(k)} & \Phi_{12}^{(k)} & \Phi_{13}^{(k)} & \Phi_{14}^{(k)} \\
* & \Phi_{22}^{(k)} & \Phi_{23}^{(k)} & \Phi_{24}^{(k)} \\
* & * & \Phi_{33}^{(k)} & \Phi_{34}^{(k)} \\
* & * & * & \Phi_{44}^{(k)}
\end{array}\right]+\sigma Z_{k}^{-1} \sigma^{\mathrm{T}} .
$$

Since $E\left(\varsigma^{\mathrm{T}} Z_{k} \varsigma\right)=E \int_{t-h(t)}^{t} \operatorname{tr}\left[g^{\mathrm{T}}(s) Z_{k} g(s)\right] \mathrm{d} s$, it follows that

$$
E L_{v=0} V(t) \leqslant \sum_{k=1}^{q} E \eta^{\mathrm{T}}(t) \xi_{k} \Phi^{(k)} \eta(t)
$$

By Schur's complement, $\Phi^{(k)}<0$ is equivalent to LMI (13). From the proof of Theorem 1 [12], there exist a scalar $\alpha$ such that

$$
\lim _{t \rightarrow \infty} \sup \frac{1}{t} \log E\|x(t)\|^{2} \leqslant-\alpha
$$

which implies that system (1) is exponentially stable in mean square. The proof of Theorem 1 is completed.

Remark 1 By constructing an output feedback controller, we can obtain the stability criteria for system with output feedback in the same methods. So, it is without loss of generality for the discussion of system (1).

Note that a delay-dependent and rate-independent exponential stability criterion for system (1) with polytopic-type uncertainties (3) and a delay satisfying (4) and (5) can be derived from Theorem 1 by choosing $Q_{k}=0$ as follows.

Corollary 1 Given scalar $\tau>0$, system (1) with polytopic-type uncertainties (3) and a time-varying delay satisfying (4) is exponentially stable in mean square if there exist symmetric positive-definite $P_{k}, Z_{k}, R_{i j}^{(k)}(k=$ $1, \cdots, q)$ and appropriately dimensioned matrices $N_{r}$ and $T_{r}(r=1,2,3)$ such that $R_{i j}^{(k)}=R_{j i}^{(k)}(i, j=1,2,3)$ and the following LMI holds for $k=1,2, \cdots, q$ :

$$
\hat{\Phi}^{(k)}=\left[\begin{array}{ccccc}
\hat{\Phi}_{11}^{(k)} & \Phi_{12}^{(k)} & \Phi_{13}^{(k)} & \Phi_{14}^{(k)} & R_{13}^{(k)} \\
* & \hat{\Phi}_{22}^{(k)} & \Phi_{23}^{(k)} & \Phi_{24}^{(k)} & R_{23}^{(k)} \\
* & * & \Phi_{33}^{(k)} & \Phi_{34}^{(k)} & 0 \\
* & * & * & \Phi_{44}^{(k)} & 0 \\
* & * & * & * & -Z_{k}
\end{array}\right]<0
$$

and

$$
R^{(k)}=\left[\begin{array}{ccc}
R_{11}^{(k)} & R_{12}^{(k)} & R_{13}^{(k)} \\
* & R_{22}^{(k)} & R_{23}^{(k)} \\
* & * & R_{33}^{(k)}
\end{array}\right]
$$

where

$$
\begin{aligned}
\hat{\Phi}_{11}^{(k)}= & N_{1} A_{k}+A_{k}^{\mathrm{T}} N_{1}^{\mathrm{T}}+T_{1} C_{k}+C_{k}^{\mathrm{T}} T_{1}^{\mathrm{T}} \\
& +\tau R_{11}^{(k)}+R_{13}^{(k)}+R_{13}^{(k) T}, \\
\hat{\Phi}_{22}^{(k)}= & N_{2} A_{d k}+A_{d k}^{\mathrm{T}} N_{2}^{\mathrm{T}}+T_{2} C_{d k}+C_{d k}^{\mathrm{T}} T_{2}^{\mathrm{T}} \\
& +\tau R_{22}^{(k)}-R_{23}^{(k)}-R_{23}^{(k) T},
\end{aligned}
$$

and $\Phi_{i j}^{(k)}(i=1,2,3 ; j=2,3,4)$ are defined in (13).

\subsection{Delay-independent rate-dependent robust expo- nential stability}

If we set the matrices $Z_{k}$ and $R_{i j}^{(k)}(i=j=1,2,3)$ to zero, then we can obtain a delay-independent and ratedependent exponential stability criterion for system (1) with polytopic-type uncertainties (3) and a time-varying delay satisfying (4) and (5). In this case, Theorem 1 becomes the following corollary.

Corollary 2 Given scalar $\mu<1$ and system (1) with polytopic-type uncertainties (3) and a time-varying delay satisfying (5) is robust exponentially stable in mean square, if there exist symmetric positive-definite matrices $P_{k}$ and $Q_{k}$ and appropriately dimensioned matrices $N_{r}$ and $T_{r}$ $(r=1,2,3,4)$ such that the following LMI holds for $k=1,2, \cdots, q$ :

$$
\tilde{\Phi}^{(k)}=\left[\begin{array}{cccc}
\tilde{\Phi}_{11}^{(k)} & \tilde{\Phi}_{12}^{(k)} & \tilde{\Phi}_{13}^{(k)} & \tilde{\Phi}_{14}^{(k)} \\
* & \tilde{\Phi}_{22}^{(k)} & \tilde{\Phi}_{23}^{(k)} & \tilde{\Phi}_{24}^{(k)} \\
* & * & \tilde{\Phi}_{33}^{(k)} & \tilde{\Phi}_{34}^{(k)} \\
* & * & * & \tilde{\Phi}_{44}^{(k)}
\end{array}\right]<0,
$$




$$
\begin{aligned}
& \text { where } \\
& \tilde{\Phi}_{11}^{(k)}=N_{1} A_{k}+A_{k}^{\mathrm{T}} N_{1}^{\mathrm{T}}+T_{1} C_{k}+C_{k}^{\mathrm{T}} T_{1}^{\mathrm{T}}+Q_{k}, \\
& \tilde{\Phi}_{12}^{(k)}=N_{1} A_{k d}+A_{k}^{\mathrm{T}} N_{2}^{\mathrm{T}}+T_{1} C_{k d}+C_{k}^{\mathrm{T}} T_{2}^{\mathrm{T}}, \\
& \tilde{\Phi}_{13}^{(k)}=P_{k}-N_{1}+A_{k}^{\mathrm{T}} N_{3}^{\mathrm{T}}+C_{k}^{\mathrm{T}} T_{3}^{\mathrm{T}} \text {, } \\
& \tilde{\Phi}_{14}^{(k)}=A_{k}^{\mathrm{T}} N_{4}^{\mathrm{T}}-T_{1}+C_{k}^{\mathrm{T}} T_{4}^{\mathrm{T}} \text {, } \\
& \tilde{\Phi}_{22}^{(k)}=N_{2} A_{k d}+A_{k d}^{\mathrm{T}} N_{2}^{\mathrm{T}}+T_{2} C_{k d}-(1-\mu) Q_{k} \\
& +C_{k d}^{\mathrm{T}} T_{2}^{\mathrm{T}} \text {, } \\
& \tilde{\Phi}_{23}^{(k)}=-N_{2}+A_{k d}^{\mathrm{T}} N_{3}^{\mathrm{T}}+C_{k d}^{\mathrm{T}} T_{3}^{\mathrm{T}}, \\
& \tilde{\Phi}_{24}^{(k)}=A_{k d}^{\mathrm{T}} N_{4}^{\mathrm{T}}-T_{2}+C_{k d}^{\mathrm{T}} T_{4}^{\mathrm{T}}, \quad \tilde{\Phi}_{33}^{(k)}=-N_{3}-N_{3}^{\mathrm{T}} \text {, } \\
& \tilde{\Phi}_{34}^{(k)}=-N_{4}^{\mathrm{T}}-T_{3}, \tilde{\Phi}_{44}^{(k)}=2 P_{k}-T_{4}^{\mathrm{T}}-T_{4} .
\end{aligned}
$$

In addition, if there is no stochastic uncertainty in system (1), that is, $\beta(t)$ is assumed to be zero, system (1) degenerate into system [10]. We obtain the following corollary.

Corollary 3 Given scalars $\tau>0$, and $\mu<1$, system (1) with $\beta(t)=0$ and with time-varying delay satisfying (4) and (5) is asymptotically stable, if there exist symmetric positive-definite matrix $P_{k}>0, Q_{k} \geqslant 0$, and $R_{i j}^{(k)}$ $(i, j=1,2,3)$ and appropriately dimensioned matrices $N_{r}$ $(r=1,2,3,4)$ such that $R_{i j}^{(k)}=R_{j i}^{(k)}$ and the following LMIs hold for $k=1,2, \cdots, q$ :

$$
\Sigma^{(k)}=\left[\begin{array}{ccc}
\Sigma_{11}^{(k)} & \Sigma_{12}^{(k)} & \Sigma_{13}^{(k)} \\
* & \Sigma_{22}^{(k)} & \Sigma_{23}^{(k)} \\
* & * & \Sigma_{33}^{(k)}
\end{array}\right]<0
$$

and

$$
R^{(k)}=\left[\begin{array}{ccc}
R_{11}^{(k)} & R_{12}^{(k)} & R_{13}^{(k)} \\
* & R_{22}^{(k)} & R_{23}^{(k)} \\
* & * & R_{33}^{(k)}
\end{array}\right]>0
$$

where

$$
\begin{aligned}
\Sigma_{11}^{(k)}= & N_{1} A_{k}+A_{k}^{\mathrm{T}} N_{1}^{\mathrm{T}}+Q_{k}+\tau R_{11}^{(k)}+R_{13}^{(k)}+R_{13}^{(k) T}, \\
\Sigma_{12}^{(k)}= & N_{1} A_{d k}+A_{k}^{\mathrm{T}} N_{2}^{\mathrm{T}}+\tau R_{12}^{(k)}-R_{13}^{(k)}+R_{23}^{(k) T}, \\
\Sigma_{13}^{(k)}= & P_{k}-N_{1}+A_{k}^{\mathrm{T}} N_{3}^{\mathrm{T}}, \\
\Sigma_{22}^{(k)}= & N_{2} A_{d k}+A_{d k}^{\mathrm{T}} N_{2}^{\mathrm{T}}-(1-\mu) Q_{k}+\tau R_{22}^{(k)} \\
& -R_{23}^{(k)}-R_{23}^{(k) T}, \\
\Sigma_{23}^{(k)}= & -N_{2}+A_{d k}^{\mathrm{T}} N_{3}^{\mathrm{T}}, \quad \Sigma_{33}^{(k)}=-N_{3}-N_{3}^{\mathrm{T}}+\tau R_{33}^{(k)} .
\end{aligned}
$$

Remark 2 Corollary 3 is equivalent to Theorem 2 [10]. Moreover, because the new Lyapunov-Krasovskii functional is different from that in [10], the results obtained from Corollary 3 are less conservative than existing ones [10].

Besides, if we assume $A_{d k}=C_{d k}=0$, system (1) turns into a stochastic system without time delay, the following corollary can be acquired.

Corollary 4 Given scalars $\tau>0$ and $\mu<1$, system (1) with time-varying delay satisfying (4) and (5) and with $A_{d k}=C_{d k}=0$ exponentially stable in mean square, if there exist symmetric positive-definite matrix $P_{k}>0$, and appropriately dimensioned matrices $N_{r}$ and $T_{r}(r=1,3,4)$ such that the following LMI holds for $k=1,2, \cdots, q$ :

$$
\hat{\Sigma}^{(k)}=\left[\begin{array}{ccc}
\hat{\Sigma}_{11}^{(k)} & \hat{\Sigma}_{13}^{(k)} & \hat{\Sigma}_{14}^{(k)} \\
* & \hat{\Sigma}_{33}^{(k)} & \breve{\Sigma}_{34}^{(k)} \\
* & * & \hat{\Sigma}_{44}^{(k)}
\end{array}\right]<0,
$$

where

$$
\begin{aligned}
& \hat{\Sigma}_{11}^{(k)}=N_{1} A_{k}+A_{k}^{\mathrm{T}} N_{1}^{\mathrm{T}}+T_{1} C_{k}+C_{k}^{\mathrm{T}} T_{1}^{\mathrm{T}}, \\
& \hat{\Sigma}_{13}^{(k)}=P_{k}-N_{1}+A_{k}^{\mathrm{T}} N_{3}^{\mathrm{T}}+C_{k}^{\mathrm{T}} T_{3}^{\mathrm{T}}, \\
& \hat{\Sigma}_{14}^{(k)}=A_{k}^{\mathrm{T}} N_{4}^{\mathrm{T}}-T_{1}+C_{k}^{\mathrm{T}} T_{4}^{\mathrm{T}}, \quad \hat{\Sigma}_{33}^{(k)}=-N_{3}-N_{3}^{\mathrm{T}}, \\
& \hat{\Sigma}_{34}^{(k)}=-N_{4}^{\mathrm{T}}-T_{3}, \quad \hat{\Sigma}_{44}^{(k)}=P_{k}-T_{4}^{\mathrm{T}}-T_{4} .
\end{aligned}
$$

Remark 3 When $\mu=0$, the delay is time invariant. From Theorem 1 and Corollary 2, we can easily obtain the delay-dependent and delay-independent robust exponential stability criteria for continuous-time linear stochastic system with polytopic-type uncertainties and with timeinvariant state delay, respectively.

\section{Numerical simulation}

In this section, for the purpose of illustrating the usefulness and flexibility of the methods in this paper, we present some simulation examples.

Example 1 Consider the following time-varying delay system $\Sigma_{1}$ with polytopic-type uncertainties ([10] Example $2)$, where

$$
A=\left[\begin{array}{cc}
0 & -0.12+12 \rho \\
1 & -0.465-\rho
\end{array}\right], \quad A_{d}=\left[\begin{array}{cc}
-0.1 & -0.35 \\
0 & 0.3
\end{array}\right]
$$

and $\|\rho\| \leqslant 0.035$ [12]. Let $\rho_{m}=0.035$ [10] and set

$$
\begin{aligned}
& A_{1}=\left[\begin{array}{ll}
0 & -0.12+12 \rho_{m} \\
1 & -0.465-\rho_{m}
\end{array}\right], \\
& A_{2}=\left[\begin{array}{ll}
0 & -0.12-12 \rho_{m} \\
1 & -0.465+\rho_{m}
\end{array}\right], \\
& A_{1 d}=A_{2 d}=A_{d}=\left[\begin{array}{cc}
-0.1 & -0.35 \\
0 & 0.3
\end{array}\right] .
\end{aligned}
$$

When $\mu=0$, the upper bound on the time delay obtained in [10] is 0.863 . However, by Corollary 3 , the system $\Sigma_{1}$ is robustly stable for delay $\tau=0.8758$, which is better than the values in [10]. Table 1 shows a comparison of the upper bounds for $\mu \neq 0$ obtained by Fridman and Shaked's method [13], He's method [10], and our methods (Corollary 3 ). It is clear that the upper bounds obtained by Corollary 3 are larger than those given in [13] and [10].

Table 1 Calculation results for Example 1.

\begin{tabular}{ccccc}
\hline$\mu$ & 0 & 0.1 & 0.9 & any $\mu$ \\
\hline Fridman [13] & 0.782 & 0.736 & 0.454 & 0.454 \\
He [10] & 0.863 & 0.786 & 0.454 & 0.454 \\
Corollary 3 & 0.8758 & 0.8041 & 0.603 & 0.4548 \\
\hline
\end{tabular}

Example 2 Consider the robust stability of the uncertain stochastic delay system $\Sigma_{2}$ with the following parameters:

$$
\begin{aligned}
& A=\left[\begin{array}{cc}
-1 & 0.3 \rho \\
1 & -1+0.3 \sigma
\end{array}\right], \quad A_{d}=\left[\begin{array}{cc}
-1 & 0.3 \rho \\
-0.6 & -1.5+0.3 \sigma
\end{array}\right], \\
& C=\left[\begin{array}{cc}
0.1 & -0.1+0.3 \rho \\
0 & 0.1+0.3 \sigma
\end{array}\right], \quad C_{d}=\left[\begin{array}{cc}
-0.2 & 0.3 \rho \\
0 & 0.1+0.3 \sigma
\end{array}\right],
\end{aligned}
$$

and $|\rho| \leqslant 1,|\sigma| \leqslant 1$.

The parameter uncertainty can be represented by a fourvertex polytope and the upper bound of the time delay $\tau$, which guarantees that the given system is exponentially sta- 
ble in mean square, as given in Table 2. Set the initial conditions as $x(0)=[0.04,0]^{\mathrm{T}}$; Figs. 1 and 2 show the state response of polytopic model with four-vertex systems (each system has two states), as $\mu=0.5, \tau_{\max }=0.5598$, and $\mu$ is any value, $\tau_{\max }=0.3893$, respectively.

Table 2 The upper bound of $\tau$ for Example 2, as a function of the bound $\mu$.

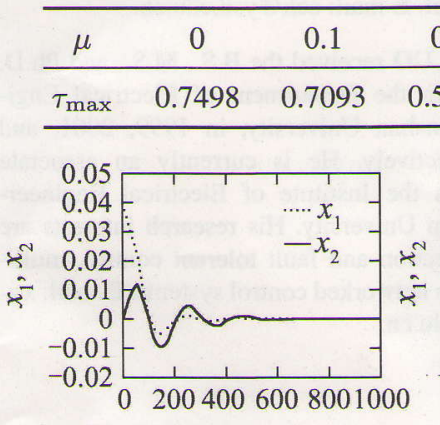

$t / \mathrm{s}$

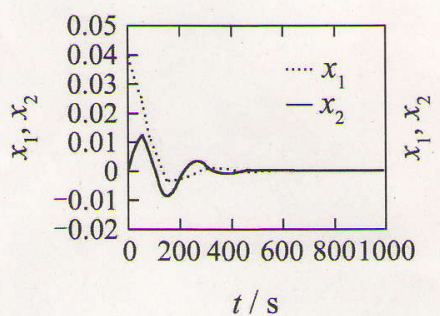

$t / \mathrm{s}$

Fig. 1 The state response of system with 4 vertices (as $\mu=0.5$, $\tau_{\max }=0.5598$ ).

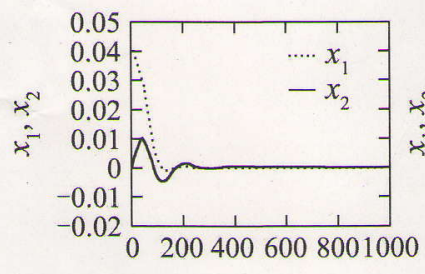

$t / \mathrm{s}$

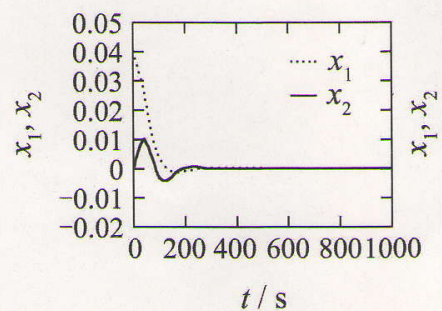

$t / \mathrm{s}$

$$
\begin{aligned}
& 0.05 \\
& 0.04 \\
\approx & 0.02 \\
= & 0.01 \\
& -0.01 \\
& -0.02
\end{aligned}
$$

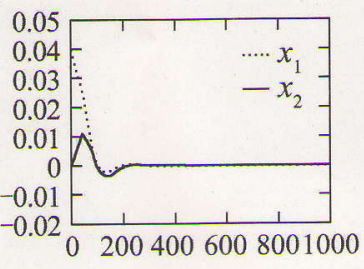

$t / \mathrm{s}$
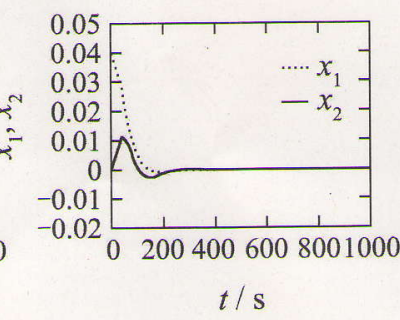

Fig. 2 The state response of system with 4 vertices (as $\mu$ is any value, $\tau_{\max }=0.3893$ ).

\section{Conclusions}

This paper presents some new stability criteria for stochastic time-varying delay systems with polytopic-type uncertainties. Based on the equivalent descriptor stochastic system and some free weighing matrices, a new type of Lyapunov-Krasovskii functional is constructed, and new techniques are developed to make the criteria less conservative. Finally, numerical examples demonstrate that the criteria presented here perform much better than the existing stable one.

\section{References}

[1] U. Haussman. Asymptotic stability of the linear Itô equation in infinite dimensions[J]. Journal of Mathematical Analysis and Applications, 1978, 65(2): $219-235$.

[2] A. Ichikawa. Stability of semilinear stochastic evolution equations[J]. Journal of Mathematical Analysis and Applications, 1982, 90(1): 12 -44 .

[3] X. Mao, N. Koroleva, A. Rodkina. Robust stability of uncertain stochastic differential delay equations[J]. Systems \& Control Letters, 1998, 35(5): 325 - 336.

[4] E. Verriest, P. Florchinger. Stability of stochastic systems with uncertain time delays[J]. Systems \& Control Letters, 1995, 24(1): 41 -47 .

[5] D. Yue, S. Won. Delay-dependent robust stability of stochastic systems with time delay and nonlinear uncertainties[J]. Electronics Letters, 2001, 37(15): 992 - 993.

[6] C. Lu, J. Tsai. An LMI-Based approach for robust stabilization of uncertain stochastic systems with time-varying delays[J]. IEEE Transactions on Automatic Control, 2003, 48(2): 286 - 289.

[7] W. Chen, Z. Guan, X. Lu. Delay-dependent exponential stability of uncertain stochastic systems with multiple delays: An LMI approach[J]. Systems \& Control Letters, 2005, 54(6): 547 - 555.

[8] C. Lu, T. Su, J. Tsai. On robust stabilization of uncertain stochastic time-delay systems-an LMI-based approach[J]. Journal of the Franklin Institute, 2005, 342(1):473 - 487.

[9] E. Fridman, U. Shaked. A descriptor system approach to $\mathrm{H}_{\infty}$ control of time-delay systems[J]. IEEE Transactions on Automatic Control, 2002, 47(2): $253-279$.

[10] Y. He, M. Wu, J. She, et al. Parameter-dependent Lyapunov functional for stability of time-delay systems with polytopic-type uncertainties[J]. IEEE Transactions on Automatic Control, 2004, 49(5): $828-832$.

[11] Y. Wang, L. Xie, C. Souza. Robust control of a class of uncertain nonlinear system[J]. Systems \& Control Letters, 1992, 19(1): 139 149

[12] Y. Li, X. Guan, D. Peng. Exponential stability criteria for uncertain stochastic systems[C]/Proceedings of the 27th Chinese Control Conference. Beijing: Beijing University of Aeronautics \& Astronautics Press, 2008: 21 - 25.

[13] E. Fridman, U. Shaked. Parameter dependent stability and stabilization uncertain time-delay systems[J]. IEEE Transactions on Automatic Control, 2003, 48(5): 861 - 866.

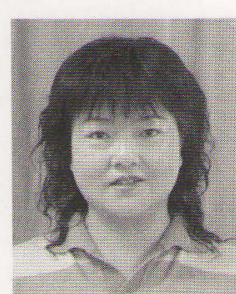

Yumei LI received the B.S. degree in Theoretical and Applied Mechanics from Lanzhou University, Lanzhou, China, and the M.S. degree in Control Theory and Control Engineering from Xinjiang University, Urumuqi, China, in 1998 and 2004, respectively. She is currently pursuing her Ph.D. degree in the Institute of Electrical Engineering, Yanshan University, Qinhuangdao, China. In July 1998, She joined the Faculty of the Department of Mathematics and System Science at Xinjiang University, Urumuqi, China, where she is currently an instructor. Her research interests are in robust control and robust filtering of stochastic systems and in distributed control and cooperative control of multiagent system. E-mail: zwzlym@163.com.

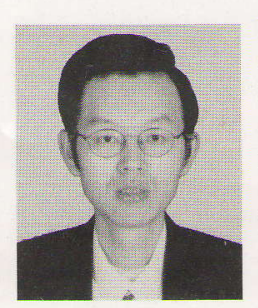

Xinping GUAN received the B.S. degree in Mathematics from Harbin Normal University, Harbin, China, the M.S. degree in Applied Mathematics, and the Ph.D. degree in Electrical Engineering, from Harbin Institute of Technology, in 1986, 1991, and 1999 , respectively. He is currently a professor and Dean of the Institute of Electrical Engineering, Yanshan University, Qinhuangdao, China. He is the (co)author of more than 100 papers in mathematical technical journals, and conferences. His current research interests are 
in time-delay systems, wireless sensor networks, congestion control of networks, robust control and in intelligent control for nonlinear systems, distributed control and cooperative control of multiagent system, chaos control, and synchronization. He is serving as an associate editor of IEEE Transaction on Systems, Man and Cybernetics (Part C) and a reviewer of Mathematic Review of America. He is National Science Fund for Distinguished Young Scholars of China, 2005. E-mail: xpguan@ysu.edu.cn.

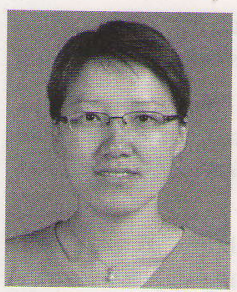

Dan PENG received the B.S. degree in Applied Mathematics from Beihua University, Jilin, China, the M.S. degree in Operational Research and Cybernetics and the Ph.D. degree in Control Theory and Control Engineering from Yanshan University, Qinhuangdao, China, in 2001, 2003, and 2008, respectively. Since June 2008, she is an instructor of the College of Science, Yanshan University, Qinhuangdao, China. Her research interests are in 2 D time-delay systems and in robust control and adaptive control. E-mail: dpeng1219@yahoo.com.cn.

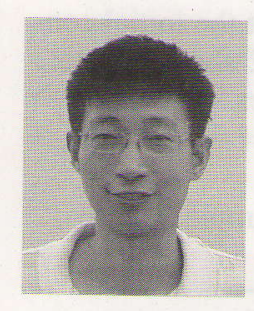

Changchun HUA received the B.S., M.S., and Ph.D. degrees in Electrical Engineering from Yanshan University, Qinhuangdao, China, in 2000, 2002 , and 2005, respectively. He is currently an associate professor in the Institute of Electrical Engineering, Yanshan University. His research interests are in nonlinear control systems, control systems design over network, teleoperation systems, and intelligent control. E-mail: cch@ysu.edu.cn.

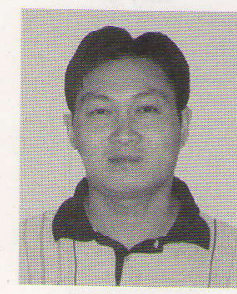

Xiaoyuan LUO received the B.S., M.S., and Ph.D. degrees from the Department of Electrical Engineering, Yanshan University, in 1999, 2001, and 2004, respectively. He is currently an associate professor in the Institute of Electrical Engineering, Yanshan University. His research interests are in fault detection and fault tolerant control, multiagent, and in networked control systems. E-mail: xyLuo@ysu.edu.cn. 